SHORT REPORT

\title{
The relation between Ashworth scale scores and the excitability of the $\alpha$ motor neurones in patients with post-stroke muscle spasticity
}

\author{
A M O Bakheit, V A Maynard, J Curnow, N Hudson, S Kodapala
}

Background: The modified Ashworth scale (MAS) is the most widely used method for assessing muscle spasticity in clinical practice and research. However, the validity of this scale has been challenged.

Objectives: To compare the MAS with objective neurophysiological tests of spasticity.

Methods: The MAS was recorded in patients with post-stroke lower limb muscle spasticity and correlated with the excitability of the $\alpha$ motor neurones. The latter was evaluated by measuring the latency of the Hoffmann reflex ( $\mathrm{H}$ reflex) and the ratio of the amplitude of the maximum $\mathrm{H}$ reflex $\left(\mathrm{H}_{\text {max }}\right)$ to that of the compound action motor potential of the soleus muscle $\left(M_{\max }\right)$.

Results: Data on 24 randomly recruited patients were analysed. Patients were divided into two groups according to their MAS score: 14 had a MAS score of 1 (group A) and 10 scored 2 (group B). The two groups were comparable with respect to age and sex, but in group $A$ there was a longer period since the stroke. The $\mathrm{H}$ reflex latency was reduced and the $H_{\max }: M_{\max }$ ratio was increased in both groups. The $H_{\max }: M_{\max }$ ratio values were higher for group $B$ but the differences were not statistically significant.

Conclusions: There is a relation between the MAS scores and $\alpha$ motor neurone excitability, although it is not linear. This suggests that the MAS measures muscle hypertonia rather than spasticity.

A ccurate evaluation of the effectiveness of the therapeutic interventions used in the management of muscle spasticity has been hampered by the lack of valid and objective outcome measures that can be applied easily and repeatedly in clinical settings. The methods that are currently used for this purpose fall into three groups: methods that assess the resistance to passive muscle stretch, neurophysiological tests, and functional outcome scales. None of these methods is entirely satisfactory.

The tests that are based on the assessment of resistance to passive muscle stretch include the modified Ashworth scale (MAS) $)^{1}$ and measurements of the joint range of motion with a goniometer or a motion analysis system. The MAS is probably the most widely used test for the measurement of muscle spasticity in research and clinical practice. However, this method suffers from important limitations. Hypertonia in patients with upper motor neurone lesions results from a combination of spasticity, thixotropy, and changes in the viscoelastic properties of muscle which may ultimately lead to the development of fixed muscle contractures. ${ }^{23}$ The MAS does not reliably distinguish between these components of hypertonia. Furthermore, the inter-rater and intra-rater reliability of this scale in assessing lower limb spasticity remains doubtful. ${ }^{4}$ Another limitation of the MAS is that the test conditions have not been standardised. For example, while some clinicians assess the muscle tone from the resting state without previous muscle stretch, others ${ }^{5}$ have recommended flexion and extension of the limb a few times immediately before the actual measurement is taken. This lack of standardisation may introduce measurement errors because the stretch reflex excitability in the resting state may be different from that of the activated muscle. ${ }^{6}$

Muscle spasticity is associated with various neurophysiological changes in the segmental spinal circuitry. These include increased excitability of the $\alpha$ motor neurones, decreased presynaptic and reciprocal inhibition, and reduced lA facilitation. ${ }^{7}$ The degree of the $\alpha$ motor neurone excitability, as measured by the Hoffmann reflex (H reflex), has been shown to correlate with the clinically observed increase in the myotatic stretch reflex activity ${ }^{8}$ and to discriminate between spasticity and normal muscle tone. ${ }^{9}$ Both the latency and the ratio of the maximum amplitude of the $\mathrm{H}$ reflex to that of the motor response (that is, the compound motor action potential) of the soleus muscle $\left(\mathrm{H}_{\max }: \mathrm{M}_{\max }\right.$ ratio $)$ have been shown to be reliable measures of the $\alpha$ motor neurone excitability. ${ }^{10}$ However, these tests have not received wide acceptance in clinical practice because they are time consuming and require special equipment and expertise. Nonetheless, they are valuable objective tools that may be useful for the validation of other less robust outcome measures of muscle spasticity.

Although most of the limitations of the MAS can be overcome, ${ }^{11}$ its method of scoring the severity of hypertonia remains problematic. This is because it depends on the examiner's subjective impression of the perceived degree of resistance to passive muscle stretch. However, it would be reasonable to disregard this weakness if the MAS can be shown to correlate closely with the objective neurophysiological tests of spasticity. Our aim in the present study was to establish whether such a correlation exists.

\section{METHODS}

Adult patients with first ever ischaemic or haemorrhagic stroke of at least six months' duration and resulting in hemiplegia were asked to take part in the study. Patients were recruited if they had a clinically detectable increase in muscle tone and were not receiving antispasticity drugs. Those with fixed muscle contractures at the ankle joint were excluded. A fixed muscle contracture was deemed to be present if the range of motion at the ankle on passive stretch was severely or fully restricted - that is, the MAS score was 4 (see below). The assessment of muscle tone and the neurophysiological tests were made in the same session. The assessment of muscle tone was always carried out first.

The study was approved by the relevant ethics committee and all participants gave their informed written consent. 


\begin{tabular}{ll} 
Table 1 & The modified Ashworth scale \\
\hline 0 & $\begin{array}{l}\text { No increase in muscle tone } \\
1\end{array}$ \\
$\begin{array}{l}\text { Slight increase in muscle tone, manifested by a catch and release or minimal resistance at the end of } \\
\text { the range of movement (ROM) when the affected part is moved in flexion or extension. }\end{array}$ \\
$1+\quad \begin{array}{l}\text { Slight increase in muscle tone, manifested by a catch followed by minimal resistance throughout the } \\
\text { remainder (less than half) of the ROM. }\end{array}$ \\
3 & $\begin{array}{l}\text { More marked increase in muscle tone through most of the ROM, but affected part easily moved. } \\
\text { Considerable increase in muscle tone, passive movement difficult. }\end{array}$ \\
4 & Affected parts rigid in flexion and extension.
\end{tabular}

\section{The modified Ashworth scale}

Spasticity of the calf muscles was assessed clinically with the MAS. As shown in table 1, the scale measures the degree of muscle hypertonia on a six point scale, ranging from 0 to $4(0$, normal muscle tone; 4 , fixed muscle contracture). Resistance to passive muscle stretch was measured at the ankle joint of the hemiplegic lower limb with the patient lying flat in the supine position. All tests were carried out by one investigator in order to eliminate inter-rater variability.

\section{The $\mathrm{H}$ reflex latency and $\mathrm{H}_{\max }: \mathrm{M}_{\max }$ ratio}

The $\mathrm{H}$ reflex and the $\mathrm{M}$ wave were acquired with the Neuromapper EMG machine (Neuroscience Ltd, Harold Hill, Essex, UK). The bandpass filter was set at $3 \mathrm{~Hz}$ to $3 \mathrm{kHz}$. The amplified signals were digitised and stored in the computer's hard disk for subsequent calculation of the $\mathrm{H}_{\max }: \mathrm{M}_{\max }$ ratio and the $\mathrm{H}$ reflex latency.

The test was carried out with the patient lying in the prone position and the feet suspended over the end of the bed and the head resting on a pillow. Bipolar silver-silver chloride surface electrodes $5 \mathrm{~mm}$ in diameter (Nicolet Biomedical, Maddison, Wisconsin, USA) were applied as follows:

Stimulating electrode (anode): over the tibial nerve in the popliteal fossa.

Active electrode: over the medial head of the gastrocnemius halfway between the medial malleolus and the tibial epicondyle.

\begin{tabular}{|c|c|c|c|c|c|}
\hline Patient & Age/sex & $\begin{array}{l}\text { Months } \\
\text { since stroke }\end{array}$ & $\begin{array}{l}\text { Ashworth } \\
\text { scale score }\end{array}$ & $\begin{array}{l}\mathrm{H} \text { reflex } \\
\text { latency }\end{array}$ & $H_{\max }: M_{\max }$ \\
\hline 1 & $70 / M$ & 8 & 1 & 36.8 & 0.37 \\
\hline 2 & $56 / M$ & 12 & 1 & 33.8 & 0.86 \\
\hline 3 & $71 / M$ & 11 & 1 & 33.2 & 0.38 \\
\hline 4 & $53 / \mathrm{F}$ & 36 & 1 & 37.6 & 0.89 \\
\hline 5 & $47 / F$ & 7 & 1 & 29.4 & 0.38 \\
\hline 6 & $53 / M$ & 14 & 1 & 33.8 & 0.74 \\
\hline 7 & $63 / F$ & 40 & 1 & 30.2 & 0.59 \\
\hline 8 & $69 / \mathrm{F}$ & 30 & 1 & 34.4 & 1.03 \\
\hline 9 & $76 / F$ & 10 & 1 & 32.2 & 0.35 \\
\hline 10 & $68 / M$ & 18 & 1 & 35.2 & 0.25 \\
\hline 11 & $76 / M$ & 8 & 1 & 29.4 & 0.17 \\
\hline 12 & $72 / M$ & 11 & 1 & 35.8 & 0.83 \\
\hline 13 & $54 / F$ & 27 & 1 & 30.6 & 0.38 \\
\hline 14 & $46 / M$ & 9 & 1 & 32.6 & 0.38 \\
\hline 15 & $55 / \mathrm{M}$ & 8 & 2 & 36.0 & 0.66 \\
\hline 16 & $46 / M$ & 2 & 2 & 32.6 & 0.30 \\
\hline 17 & $46 / M$ & 11 & 2 & 35.4 & 0.68 \\
\hline 18 & $52 / M$ & 11 & 2 & 33.2 & 0.40 \\
\hline 19 & $66 / M$ & 10 & 2 & 32.0 & 0.43 \\
\hline 20 & $64 / F$ & 6 & 2 & 33.8 & 0.55 \\
\hline 21 & $55 / \mathrm{M}$ & 4 & 2 & 33.2 & 0.71 \\
\hline 22 & $81 / M$ & 12 & 2 & 35.0 & 0.60 \\
\hline 23 & $70 / \mathrm{F}$ & 30 & 2 & 36.2 & 0.40 \\
\hline 24 & $56 / M$ & 8 & 2 & 37.8 & 1.11 \\
\hline 25 & $31 / M$ & 5 & 3 & 34.0 & 1.15 \\
\hline
\end{tabular}

$F$, female; $H_{\max }$ maximum amplitude of the $H$ reflex; $M$, male; $M_{\max ,}$ maximum amplitude of the motor response.
Earth electrode: over the lateral head of the gastrocnemius muscle between the stimulator and the active electrode.

Reference electrode: over the insertion of the Achilles tendon.

Before the application of the electrodes, the skin was cleansed until a skin impedance of less than $10 \mathrm{kohm}$ was obtained. The tibial nerve was stimulated with a rectangular electrical pulse of $\mathrm{l} \mathrm{ms}$ duration and with a stimulus frequency of 1 per five seconds. The stimulation procedure described by Braddom and Johnson ${ }^{12}$ was followed. Initially the optimal position for stimulating the tibial nerve in the popliteal fossa was determined by moving the stimulating electrode around until a visible contraction of the gastrocnemius muscle was seen. Then the current was gradually increased until an $\mathrm{H}$ reflex without an $M$ response was recorded. The response with the largest amplitude was selected as the $\mathrm{H}_{\max }$ and other values were rejected. The stimulus intensity was then increased further in small increments until the maximum $\mathrm{M}$ response was obtained. The $\mathrm{H}$ reflex was identified as a triphasic wave with a small initial positive deflection followed by a larger negative one.

The maximum amplitudes of the $\mathrm{H}$ reflex and the $\mathrm{M}$ wave were measured from the peak of the positive to the peak of the negative deflections. The $\mathrm{H}_{\max }$ to $\mathrm{M}_{\max }$ ratio was calculated by dividing the maximum amplitudes of the $\mathrm{H}$ reflex by that of the $\mathrm{M}$ wave. The $\mathrm{H}$ reflex latency was measured from the start of the stimulation to the onset of the initial deflection of the $\mathrm{H}$ reflex.

\section{RESULTS}

Twenty five consecutive stroke patients who fulfilled the study entry criteria completed the evaluation. Patients were grouped according to the severity of the spasticity, as measured by the MAS. Fourteen patients scored 1 on the MAS (group A) and 10 scored 2 (group B). Only one subject had a MAS score of 3 and was excluded from further analysis. Details of the patients' demographic and clinical data are given in table 2 .

The mean ages (range) of patients in groups A and B were 62.4 (46 to 72 ) years and 59.1 (46 to 81 ) years, respectively. There were eight men in each group. Patients in group A had a mean period since the stroke onset of 17.2 months, compared with 10.2 months in group B. The mean (SD) $\mathrm{H}$ reflex latencies for groups A and B were 33.2 (2.6) ms and 34.5 (1.8) ms, respectively. The difference between the groups was not statistically significant $(t=-1.32, \mathrm{df} 22, \mathrm{p}=0.2)$. Analysis of the $H_{\max }: M_{\max }$ ratio showed that the mean (SD) values for group A were 0.55 (0.27) and for group B, 0.58 (0.23). The differences between groups A and B were also not statistically significant $(t=-0.32$, df $22, \mathrm{p}=0.7)$.

\section{DISCUSSION}

We set out to establish if there is a correlation between the scores of the MAS and the excitability of the $\alpha$ motor neurone in subjects with untreated muscle spasticity. The validity of the MAS as a clinical tool for assessing muscle spasticity would be enhanced if such a correlation can be established. This is because increased $\alpha$ motor neurone excitability is an 
important mechanism of muscle spasticity. ${ }^{7}$ The excitability of the $\alpha$ motor neurone was assessed in this study by measuring the $\mathrm{H}_{\max }: \mathrm{M}_{\max }$ ratio and the $\mathrm{H}$ reflex latency. The sensitivity of these tests has been confirmed previously. ${ }^{7{ }^{13}{ }^{14}}$ Hiersemenzel et al have demonstrated three stages of adaptational change in the excitability of the spinal neuronal circuits in patients with spinal cord injury. ${ }^{15}$ They found that, although the $\mathrm{H}$ reflex can be elicited shortly after the event, the $H_{\max }: M_{\max }$ ratio reached its maximum in 8 to 24 weeks and remained stable thereafter. It was therefore important to examine patients at least six months after disease onset, as we did in the present study.

Our findings confirm an increase in the mean values of the $\mathrm{H}_{\max }: \mathrm{M}_{\max }$ ratio in a random sample of patients with mild to moderately severe spasticity. However, as reported previously, ${ }^{7}$ there were marked variations between individuals with the same degree of hypertonia. Interestingly, the $\mathrm{H}_{\max }: \mathrm{M}_{\max }$ ratio was higher in the group of patients who scored 2 on the MAS than in those who had a score of 1 . However, the differences between the groups did not reach statistical significance. The study also showed a reduced latency of the $\mathrm{H}$ reflex in both groups and this was consistent with the observations of other investigators. ${ }^{13}$ Various factors could account for the poor correlation between the $\mathrm{H}_{\max }: \mathrm{M}_{\max }$ ratio and the MAS scores, as follows.

First, it has been suggested that the F wave is a more sensitive neurophysiological indicator of spasticity than the $\mathrm{H}$ reflex. ${ }^{13}{ }^{16}$ We did not record the $\mathrm{F}$ wave in our patients, and the discrepancy between the severity of muscle spasticity and the measured $\alpha$ motor neurone excitability could have reflected this. However, this explanation is unlikely because the activity of only a very small percentage of the $\alpha$ motor neurone population accounts for the occurrence of $\mathrm{F}$ wave. ${ }^{17}$

Second, the neurophysiological mechanisms that underlie spasticity include changes in the excitability of the spinal interneurones as well as the $\alpha$ motor neurone. ${ }^{7}$ Consequently, it is reasonable to assume that the study of the correlation between the changes in the spinal segmental circuitry and muscle spasticity should include recording the flexor reflexes. However, a recent study of the evolution of the neurophysiological changes in the spinal segmental circuitry ${ }^{15}$ has shown that the amplitude of flexor reflexes may fall as spasticity becomes established. This casts doubt on the clinical value of measuring the flexor reflex amplitudes in the chronic phase of spasticity. Nonetheless, the study of the correlation of long latency reflexes with spasticity remains an important question that needs to be addressed in future studies.

Finally, the poor correlation between the results of the neurophysiological tests and the degree of spasticity may also be caused by problems inherent to the MAS itself. This scale, which relies on the subjective judgement of the examiner, measures resistance to passive muscle stretch. This resistance often reflects a combination of spasticity, thixotropy, and fixed muscle contractures. ${ }^{23}$ Although in this study we took care to exclude patients with clinically detectable fixed contractures, it is not possible to be certain that changes in the fibre structure or the viscoelastic properties of muscle were not present.

\section{Conclusions}

Our study has shown a shorter $\mathrm{H}$ reflex latency and an increase in the $\mathrm{H}_{\max }: \mathrm{M}_{\max }$ ratio in patients with a score of 1 or 2 on the MAS. However, it failed to confirm a linear relation between these variables. The lack of a linear relation between the MAS and the $\mathrm{H}_{\max }: \mathrm{M}_{\max }$ ratio could reflect the fact that patients with only two grades of hypertonia were recruited for this study. The inclusion of patients with at least three different grades on the MAS would have allowed us to draw more precise conclusions. Nonetheless, the weak correlation between the two methods suggests that the MAS is a poor measurement scale of muscle spasticity as defined by the above neurophysiological tests.

\section{Authors' affiliations}

A M O Bakheit, Peninsula Medical School and Plymouth Primary Care Trust, Plymouth, UK

V A Maynard, Institute of Health Studies, University of Plymouth J Curnow, Department of Medical Physics, Derriford Hospital, Plymouth N Hudson, S Kodapala, Department of Neurophysiology, Derriford Hospital, Plymouth

Competing interests: none declared

Correspondence to: Professor Magid Bakheit, Beauchamp Centre, Mount Gould Hospital, Plymouth PL4 7QD, UK;

magid.bakheit@pcs-tr.swest.nhs.uk

Received 29 August 2002

In revised form 14 November 2002

Accepted 16 January 2003

\section{REFERENCES}

1 Bohannon RW, Smith MB. Interrater reliability of modified Ashworth scale of muscle spasticity. Phys Ther 1987;67:206-7.

2 Deitz V, Quintern J, Berger W. Electrophysiological studies of gait in spasticity and rigidity: evidence that altered mechanical properties of muscles contribute to hypertonia. Brain 1981;104:431-49.

3 Vattanasilp W, Ada L, Crosbie J. Contribution of thixotropy, spasticity, and contracture to ankle stiffness after stroke. J Neurol Neurosurg Psychiatry 2000;69:34-9

4 Hass BM, Bergstrom E, Jamous A, et al. The inter-rater reliability of the original and of the modified Ashworth scale for the assessment of spasticity in patients with spinal cord injury. Spinal Cord 1996;34:560-4.

5 Hufschmidt A, Mauritz K. Chronic transformation of muscle in spasticity: a peripheral contribution to increased tone. J Neurol Neurosurg Psychiatry 1985;48:676-85.

6 Ibrahim IK, Berger W, Trippel M, et al. Stretch-induced electromyographic activity and torque in spastic elbow muscles. Differential modulation of reflex activity in passive and active motor tasks. Brain 1993;1 16:971-89.

7 Delwaide PJ. Electrophysiological testing of spastic patients: its potential usefulness and limitations. In: Delwaide PJ, Young RR, eds. Clinical neurophysiology of spasticity. Contribution to assessment and pathophysiology. Amsterdam: Elsevier, 1985: 185-203.

8 Ongerboer de Visser BW, Bour L, Koelman JHTM, et al. Cumulative vibratory indices and the $\mathrm{H} / \mathrm{M}$ ratio of the soleus $\mathrm{H}$-reflex: a quantitative study in control and spastic subjects. Electroencephalogr Clin Neurophysiol 1989;73:162-6.

9 Bour LJ, Ongerboer de Visser BW, Koelman JHTM, et al. Soleus H-reflex tests in spasticity and dystonia: a computerized analysis. J Electromyogr Kinesiol 1991;1:9-19.

10 Angel RW, Hoffmann WW. The $\mathrm{H}$ reflex in normal, spastic and rigid subjects. Arch Neurol 1963;8:591-6.

11 Pandyan AD, Price CIM, Curless RH, et al. A review of the properties and limitations of the Ashworth and modified Ashworth scales as measures of spasticity. Clin Rehabil 1999;13:373-83.

12 Braddom RI, Johnson EW. Standardisation of H-reflex and diagnostic use in S1 radiculopathy. Arch Phys Med Rehabil 1974;55:161-6.

13 Joodaki MR, Olyaei GR, Bagheri $H$. The effects of electrical stimulation of the lower extremity on $\mathrm{H}$-reflex and $\mathrm{F}$-wave parameters. Electromyogr Clin Neurophysiol 2001:41:21-8.

14 Rosche J, Rub K, Niemann-Delius B, et al. Effects of physiotherapy on F-wave amplitude in spasticity. Electromyogr Clin Neurophysiol 1996;36:509-11

15 Hiersemenzel L-P, Curt A, Dietz V. From spinal shock to spasticity. Neuronal adaptations to spinal cord injury. Neurology 2000;54:1574-82.

16 Milanov GI. A comparison of methods to assess the excitability of lower motorneurones. Can J Neurol Sci 1992;19:64-8.

17 Dengler R, Kossev A, Wohlfahrt K, et al. F waves and motor unit size. Muscle Nerve 1992;15:1138-42. 